Discrete Comput Geom 32:317-338 (2004)

DOI: $10.1007 / \mathrm{s} 00454-004-2878-4$

\title{
Relaxation, New Combinatorial and Polynomial Algorithms for the Linear Feasibility Problem
}

\author{
Ulrich Betke \\ Fachbereich Mathematik, Universität Siegen, \\ D-57068 Siegen, Germany \\ betke@mathematik.uni-siegen.de
}

\begin{abstract}
We consider the homogenized linear feasibility problem, to find an $x$ on the unit sphere satisfying $n$ linear inequalities $a_{i}^{T} x \geq 0$. To solve this problem we consider the centers of the inspheres of spherical simplices, whose facets are determined by a subset of the constraints. As a result we find a new combinatorial algorithm for the linear feasibility problem. If we allow rescaling, this algorithm becomes polynomial. We point out that the algorithm also solves the more general convex feasibility problem. Moreover, numerical experiments show that the algorithm could be of practical interest.
\end{abstract}

\section{Introduction}

A central problem in optimization is the linear feasibility problem $\mathrm{F}$. An instance $A$ of $\mathrm{F}$ is to find for $n$ vectors $a_{i} \in \mathbb{Z}^{d}, i=1, \ldots, n$, and $n$ integers $b_{i}, i=1, \ldots, n$,

$$
x \in \mathbb{R}^{d} \quad \text { such that } \quad a_{i}^{T} x \geq b_{i}, \quad i=1, \ldots, n,
$$

or to show that such an $x$ does not exist.

The size $L$ of an instance $A$ is its encoding length, the amount of space to write down the problem (see the books [5], [9] and [12], which provide the background for this paper).

For an instance $A$ of $\mathrm{F}$ the set $P_{A}$ of feasible points

$$
P_{A}=\left\{x \in \mathbb{R}^{d} \mid a_{i}^{T} x \geq b_{i}, i=1, \ldots, n\right\}
$$

is a polyhedron.

The main application of $\mathrm{F}$ is the linear optimization problem to minimize a linear function over $P_{A}$. It is well known how to solve an instance of the linear optimization problem by solving one or more instances of $\mathrm{F}$. The linear feasibility problem is a 
particular case of the convex feasibility problem. Here we want to find a point in a convex set $K$. Clearly the problem depends on the representation of $K$. A very general way of representing $K$ is by a separation oracle [5]. For every $x \in \mathbb{R}^{d}$ this oracle either confirms that $x \in K$ or gives a hyperplane separating $x$ from $K$. A survey on algorithms for the convex feasibility problem is [2].

There are numerous algorithms for F. Well known algorithms are phase I of the simplex algorithm, Khachiyan's algorithm [7] and Karmarkar's algorithm [6]; for a fairly recent survey of Karmarkar's algorithm see, e.g. [10].

A class of algorithms for which the worst case behavior is not too bad are the polynomial algorithms. We say that an algorithm is (time)-polynomial if there is a polynomial $p$ such that, for all instances with size less than or equal to $L$, the algorithm gives a solution in a time at most $p(L)$. For details see again [5], [9] and [12]. Khachiyan's and Karmarkar's algorithms are polynomial while the simplex algorithm is not.

A different class of algorithms are combinatorial algorithms. We say that an algorithm for the linear feasibility problem or LP is combinatorial if, for each instance, there is a finite set of points, where each point is determined by a subset of the constraints with cardinality at most the dimension of the problem, such that all iterates are in this set. The standard example is the simplex algorithm, where all iterates are basic solutions which are determined by a subset whose cardinality is exactly the dimension of the instance. Here we present polynomial as well as combinatorial algorithms.

For our purposes Khachiyan's algorithm and an algorithm given by Agmon [1] and Motzkin and Schoenberg [8] are of particular interest as they are relaxation algorithms for $\mathrm{F}$ which may be described by the following simple prototype:

\section{Algorithm 1.1.}

1. Choose an arbitrary $x^{0}$.

2. Choose a "simple" convex set $C_{k}$ and $x^{k} \in C_{k}$ depending on $x^{k-1}$ and other data obtained in the course of the algorithm, such that $P_{A} \subset C_{k}$ and $x^{k}$ are in some way "better" than $C_{k-1}, x^{k-1}$. If $x^{k} \in P_{A}$ terminate.

3. Set $k:=k+1$ and goto step 2

In the case of Khachiyan's algorithm, $C_{k}$ is an ellipsoid and $x^{k}$ is its center. The next ellipsoid is constructed in the following way. Choose a constraint $i$ such that $a_{i}^{T} x^{k}<b_{i}$. Then the next ellipsoid $C_{k+1}$ is the smallest ellipsoid circumscribed to

$$
C_{k} \cap\left\{x \mid a_{i}^{T} x \geq a_{i}^{T} x^{k}\right\} .
$$

For an instance $A$ of $\mathrm{F}$ and $x \notin P_{A}$ we say that $i \in\{1, \ldots, n\}$ is a most violated constraint if $a_{i}^{T} x-b_{i}=\min \left\{a_{j}^{T} x-b_{j} \mid j=1, \ldots, n\right\}$. In the Agmon-MotzkinSchoenberg algorithm a most violated constraint, $a_{i}^{T} x \geq b_{i}$, say, for $x^{k}$ is chosen and $x^{k+1}$ is the projection of $x^{k}$ on the half-space $\left\{a_{i}^{T} x \geq b_{i}\right\}$. This algorithm is a very special case of an algorithm in [3].

Furthermore, the simplex algorithm for a problem in standard form may be viewed as a relaxation algorithm for the dual linear feasibility problem, where each iterate $x^{k}$ is determined by the requirement that it satisfies $d$ of the inequalities in (1) with equality. 
Further, we remark that Khachiyan's algorithm solves the problem of constructing a point in a convex set given by a separation oracle. We return to this topic after the presentation of our algorithms.

We proceed as follows: In Section 2 we present a relaxation algorithm for the homogenized form of $\mathrm{F}$ and determine its properties. In Section 3 we use additional transformations of the problem to construct a polynomial algorithm for F. Moreover, we shortly discuss its relation to the convex feasibility problem. While in the previous sections we have concentrated on the geometric aspects of the algorithms, in Section 4 we discuss the underlying linear algebra and present the results of some numerical experiments.

\section{Algorithms}

In a certain way we want to combine the algorithm of Agmon-Motzkin-Schoenberg with Khachiyan's algorithm. This means that we want to use a circumscribed body of $P_{A}$ and a point, which we may consider as a projection on this body. Furthermore, we want this body as closely related to $P_{A}$ as possible. A rather obvious choice is a simplex bounded by $d+1$ of the hyperplanes $\left\{a_{i}^{T} x=b_{i}\right\}$ and the center of its inball, as this point may be considered a simultaneous projection on all half-spaces. For several reasons this does not work very well in Euclidean space and thus we first use the well known process of homogenization to transform the problem into a spherical problem.

To proceed we need some more notation. We write $B(c, r)=\left\{x \in \mathbb{R}^{d} \mid\|x-c\| \leq r\right\}$ for the ball with center $c$ and radius $r, S(c, r)=\left\{x \in \mathbb{R}^{d} \mid\|c-x\|=r\right\}$ for the sphere bounding $B(c, r)$ and $S^{d-1}=S(0,1)$ for the unit sphere in $\mathbb{R}^{d}$. Then we define the homogenization

$$
\Phi: \mathbb{R}^{d} \rightarrow S^{d}, \quad \Phi\left(x_{1}, \ldots, x_{d}\right)=\left(x_{1}, \ldots, x_{d}, 1\right) /\left\|\left(x_{1}, \ldots, x_{d}, 1\right)\right\| .
$$

For $x=\left(x_{1}, \ldots, x_{d+1}\right) \in \mathbb{R}^{d+1}$ we denote by $\bar{x}=\left(x_{1}, \ldots, x_{d}\right)$ its projection on $\mathbb{R}^{d}$. For an instance $A$ of $\mathrm{F}$ we have for the set of feasible points $P_{A}$,

$$
\Phi\left(P_{A}\right)=\left\{x \in S^{d} \mid a_{i}^{T} \bar{x}-b_{i} x_{d+1} \geq 0, i=1, \ldots, n, x_{d+1}>0\right\} .
$$

Thus we obtain an algorithm for the linear feasibility problem $\mathrm{F}$ if we have an algorithm for the spherical feasibility problem $\mathrm{F}^{\prime}$, where an instance $A$ of $\mathrm{F}^{\prime}$ is to find

$$
x \in S^{d} \quad \text { such that } \quad a_{i}^{T} x \geq 0, \quad i=1, \ldots, n,
$$

or to show that such an $x$ does not exist. For an instance $A$ of $\mathrm{F}^{\prime}$ we denote again by $P_{A}$ the set of its feasible points.

To study $\mathrm{F}^{\prime}$ it is helpful to use the notion of violation. For an instance $A$ of $\mathrm{F}^{\prime}$ and $x \in S^{d}$ we define the violation $v(x)$ by

$$
v(x)=\max \left\{0, \max \left\{-a_{i}^{T} x \mid i=1, \ldots, n\right\}\right\} .
$$

Thus a point is feasible for an instance $A$ of $\mathrm{F}$ if and only if its violation is zero.

For our algorithms it is somehow more appropriate to take a polar point of view, i.e. we work with the normals $a_{i}$ rather than with the half-spheres $a_{i}^{T} x \geq 0$. Moreover, we 
construct a sequence of points which show that the instance is close to being infeasible. To be more precise we denote the origin of $\mathbb{R}^{d+1}$ by $O$, the convex hull, respectively affine hull, of a set $M \subset \mathbb{R}^{d+1}$ by conv $M$, respectively aff $M$, and define

Definition 2.1. Let $Q=\left\{x^{i} \in \mathbb{R}^{d+1} \mid i=1, \ldots, k\right\}$ be a set of points. We say that $Q$ is positively spanning if $Q$ is affinely independent and $O$ is contained in the relative interior of conv $Q$.

If $k=d+1$, then $Q$ is a positive basis. Here we also consider the case that $\operatorname{dim}(\operatorname{aff} Q)<d+1$.

Definition 2.2. Let $Q=\left\{x^{i} \in \mathbb{R}^{d+1} \mid i=1, \ldots, k\right\}$ be a set of points. We say that $Q$ is nearly positively spanning if $Q$ is affinely independent and the orthogonal projection $O^{\prime}$ of $O$ on aff $Q$ is contained in the relative interior of conv $Q$. The distance of $O^{\prime}$ to the origin is called the deficiency of $Q$ and is denoted by $\operatorname{def} Q$.

Thus a nearly positively spanning set is positively spanning if and only if its deficiency is zero. Moreover, for a nearly positively spanning set the deficiency is equal to the distance of the origin to its convex hull.

The evident significance of positively spanning sets for $\mathrm{F}^{\prime}$ is given by

Proposition 2.3. Let an instance $A$ of $F^{\prime}$ be given and let $Q \subset\left\{a_{1}, \ldots, a_{n}\right\}$ be $a$ positively spanning set. Then every $x \in P_{A}$ satisfies $a_{i}^{T} x=0$ for all $a_{i} \in Q$.

If the cardinality of $Q$ is $d+2$, then $A$ is infeasible. In the other case $P_{A}$ is contained in the plane $\left\{a_{i}^{T} x=0 \mid a_{i} \in Q\right\}$ and thus the dimension of the instance is reduced. In this case we may change the problem slightly in a way that $Q$ is transformed into a nearly positively spanning set with positive deficiency without changing the feasibility or infeasibility of the instance. This is quite analogous to cases of degeneration in Khachiyan's algorithm and is not discussed further (but see the remarks after Lemma 3.7).

If the points of $Q$ are contained in $S^{d}$, then nearly positively spanning sets can be nicely characterized.

Definition 2.4. Let $Q$ be a set of affinely independent points. The sphere $S(C, R)$ is said to be touching for $Q$ if $C \in$ aff $Q$ and $Q \subset S(C, R)$.

$S(C, R)$ is a circumsphere of $Q$ if $Q \subset B(C, R)$ and there is no $S\left(C^{\prime}, R^{\prime}\right)$ with this property and $R^{\prime}<R$.

Clearly, every set of affinely independent points has a unique touching sphere. Moreover, we observe that it is easy to compute a touching sphere for a given $Q$. The relation of touching sphere and circumsphere is given by the observation that a touching sphere $S(C, R)$ of $Q$ is a circumsphere if and only if $C \in \operatorname{conv} Q$.

The relation of touching spheres and nearly positively spanning sets is given by

Proposition 2.5. Let $Q \subset S^{d}$ be affinely independent, and let $S(C, R)$ be its touching sphere. Then $Q$ is nearly positively spanning if and only if $C$ is contained in the relative interior of conv $Q$. In this case $\operatorname{def} Q=\sqrt{1-R^{2}}$. 
By means of nearly positively spanning sets we may describe an algorithm for $\mathrm{F}^{\prime}$ :

\section{Algorithm 2.6.}

Input: A set $\left\{a_{1}, \ldots, a_{n} \mid a_{i} \in S^{d}\right\}$.

Output: $x \in S^{d}$ such that $a_{i}^{T} x \geq 0$ for $i=1, \ldots, n$ or a subset $\left\{a_{i_{1}}, \ldots, a_{i_{k}}\right\}$,

$k \geq 2$, such that every feasible $x$ must satisfy $a_{i_{j}}^{T} x=0$ for $j=1, \ldots, k$.

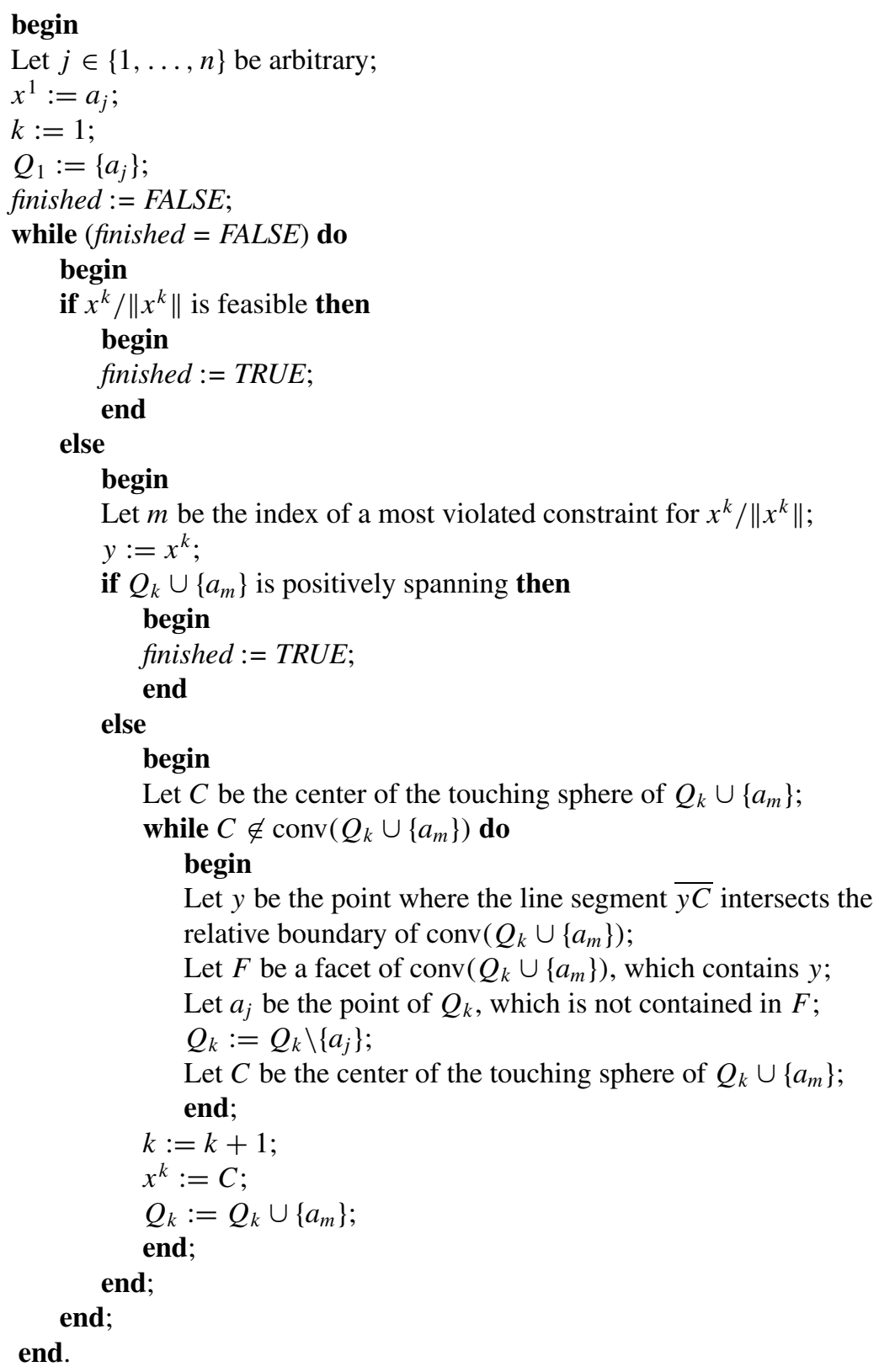


The iterates $x^{k}$ of the algorithm are the centers of touching spheres of the subsets $Q_{k}$ of $\left\{a_{1}, \ldots, a_{n}\right\}$. Clearly there are only finitely many such subsets and each subset has cardinality less than or equal to $d$. Thus the algorithm is a combinatorial algorithm as described in the Introduction. We have that the deficiency of the $Q_{k}$ is strictly decreasing and the sets $Q_{k}$ cannot be repeated. Thus we have

Lemma 2.7. Algorithm 2.6 solves $F^{\prime}$ for feasible as well as infeasible problems in finitely many steps.

As is easy to see and is discussed in more detail in the next section the iterates $x^{k}$ are the incenters of the simplices $\left\{x \in S^{d} \mid a_{i}^{T} x \geq 0, a_{i} \in Q_{k}\right\}$. As mentioned before there is a Euclidean analogue of this algorithm. While we have no quantitative information on this algorithm, it shows the close relation of our concepts to the dual simplex algorithm. In the dual simplex algorithm we consider the set $X$ of intersections of $d$ hyperplanes given by the constraints. $X$ is ordered by $c^{t} x, x \in X$, for a suitable $c \in \mathbb{R}^{d}$ and searched until a feasible point is found. In the Euclidean algorithm we consider the set $X$ of incenters of simplices defined by the intersection of $d+1$ half-spaces, given by the constraints. $X$ is ordered by the inradii of the simplices and searched with the help of this ordering.

In contrast to the simplex algorithm and the Euclidean insphere algorithm mentioned above we may modify Algorithm 2.6 slightly to obtain quantitative information on its progress.

Algorithm 2.8. Initialize the point $y$ in the outer while loop of the algorithm by choosing $y$ as the point on the line $\overline{x^{k} a_{m}}$, which is closest to the origin rather than $x$.

Lemma 2.9. Algorithm 2.8 solves the feasibility problem $F^{\prime}$ and it holds that

$$
\operatorname{def} Q_{k+1} \leq \sqrt{\frac{1-v\left(x^{k}\right)^{2}}{1+\left(\operatorname{def} Q_{k}\right)^{2}+2 \operatorname{def} Q_{k} v\left(x^{k}\right)}} \operatorname{def} Q_{k} .
$$

Proof. The $y$ used to initialize the outer while loop of Algorithm 2.8 has the distance from the origin given by the right side of (5) and by construction the distance of $x^{k+1}$ from the origin is certainly not greater than the distance of $y$ from the origin.

While the estimate (5) is not linear, it is independent of the dimension and in particular of the size of the problem. The formula may be used to estimate the number of steps that the algorithm needs to reach a given level.

Lemma 2.10. In Algorithm 2.8 it holds that $\operatorname{def} Q_{k} \leq t^{-1}$ after at most $t^{2}$ steps.

Proof. It is somewhat easier to consider the quantities $y_{k}=\left(\operatorname{def} Q_{k}\right)^{-1}$ instead of $\operatorname{def} Q_{k}$. We have from Lemma 2.9 and using $v\left(x^{k}\right) \geq 0$,

$$
y_{k+1}^{2} \geq y_{k}^{2}+1 \text {. }
$$

Together with $y_{1}=1$ this gives inductively $y_{k+1}^{2} \geq k+1$. 
It is not known (and was not investigated) whether the sequence of iterates in Algorithms 2.6 and 2.8 can differ for the same problem. According to Lemma 2.9 Algorithm 2.8 takes large steps if the deficiency or the violation are large, in particular this is the case at the start of the algorithm.

We do not have information on the global behavior of the algorithm beyond Lemma 2.10. We may obtain some more insight into its working by analyzing one step of Algorithm 2.6. Using an appropriate system of coordinates we may assume that $Q_{k}$ spans the affine plane $H=\left\{\left(x_{1}, 0, \varepsilon\right) \mid x_{1} \in \mathbb{R}^{l}\right\}$ for some fixed $\varepsilon=\operatorname{def} Q_{k}>0$ and appropriate $l$. Then we have $x^{k}=(0,0, \varepsilon)$. Further, we have that the constraint which is chosen in step 2 has the form $a_{m}=\left(x_{1}, x_{2}, \varepsilon+\eta\right)$ with $x_{1} \in \mathbb{R}^{l}, x_{2} \in \mathbb{R}^{d-l}$ and $\eta<-\varepsilon$. We find for the center $C$ of the touching sphere of $Q_{k} \cup\left\{a_{m}\right\}$ in step 4 that

$$
C=(0,0, \varepsilon)+\frac{-\eta \varepsilon}{\left\|x_{2}\right\|^{2}+\eta^{2}}\left(0, x_{2}, \eta\right) .
$$

From this we find $\|C\| \approx\left\|x^{k}\right\|$ if $|\eta| \ll\left\|x_{2}\right\|$. In this case the algorithm can only increase the cardinality of $Q_{k}$, but not significantly decrease def $Q_{k}$. Clearly the crucial point of the algorithm is whether we may expect $C \in \operatorname{conv}\left(Q_{k} \cup\left\{a_{m}\right\}\right)$. To see what happens, we denote by $a_{m}^{\prime}$ the orthogonal projection of $a_{m}$ onto $H$. By construction we have that $x^{k}$ is the orthogonal projection of $C$ onto $H$. Finally, let $y \in \operatorname{relbd}\left(\operatorname{conv} Q_{k}\right.$ ) be the point that $x^{k} \in \overline{y a_{m}^{\prime}}$. Now an examination of the triangle $y, a_{m}^{\prime}, a_{m}$ shows that $C \notin \operatorname{conv}\left(Q_{k} \cup\left\{a_{m}\right\}\right)$ if $\left\|y-x^{k}\right\| \ll\left\|a_{m}^{\prime}-x^{k}\right\|$. Clearly this may happen and in a single step the algorithm may make very little progress. Fortunately this is not the complete story.

In the algorithm we gather information about $a_{i}$. If we define for $x \in S^{d}$,

$$
M(x)=\left\{a \in S^{d} \mid a^{T} x \geq-v(x)\right\},
$$

then we have for every iterate $x^{j}$ that $a_{i} \in M\left(x^{j}\right), i=1, \ldots, n$. Consequently all $a_{i}$ satisfy for all $k$,

$$
a_{i} \in \bigcap_{j=1}^{k} M\left(x^{j}\right) .
$$

Unfortunately the sets $M\left(x^{k}\right)$ are not convex and this information is hard to use.

If we want to repeat the bad step from above, the set $M\left(x^{k}\right)$ tells us that either for the new $\varepsilon^{\prime}, \eta^{\prime}$ it holds that $\left|\varepsilon^{\prime}+\eta^{\prime}\right| \ll|\varepsilon+\eta|$ or the angle between $a_{m}$ and $a_{m^{\prime}}$ must be large. Thus we can hope that bad steps cannot be repeated too often. A precise analysis of the phenomena appears to be difficult.

\section{A Polynomial Algorithm}

In view of the last remark in the previous section it is natural to use periodically a rescaling to increase the deficiency in order to speed up the convergence. While it is simple to give a transformation of the sphere which takes polyhedra to polyhedra and increases the deficiency of a set $Q_{k}$, it appears difficult to work out the consequences of iterating such transformations. 
Fortunately it turns out that, for $\operatorname{def}\left(Q_{k}\right)$ sufficiently small, it is possible to find a transformation which increases the volume of the sets $P_{A}$. To work out our ideas it is necessary to recall some concepts of spherical convexity. A set $M \subset S^{d}$ is called convex if there exists a half-sphere which contains $M$ and for every pair $x, y \in M$ there exists a great circle $C$ such that $C \cap M$ is connected. The intersection of convex sets is itself convex. By this we may define, for every $M \subset S^{d}$ which is contained in a half-sphere, the spherical convex hull co $M$ as the intersection of all convex subsets of $S^{d}$ which contain $M$. Here we have chosen the notation co $M$ to distinguish the spherical convex hull from the Euclidean convex hull used in the previous section. As every half-sphere is convex we have that the intersection of half-spheres is convex. We say that the intersection of finitely many half-spheres is a (spherical) polyhedron. An example of a spherical polyhedron is the set $P_{A}$ of feasible points of an instance $A$ of problem $\mathrm{F}^{\prime}$.

We denote for two points $x, y \in S^{d}$ the spherical distance by $\angle(x, y)$, i.e. $\cos (\angle(x, y))$ $=x^{T} y$. Next for $x \in S^{d}$ and $0 \leq \rho \leq \pi$,

$$
K_{\rho}(x)=\left\{y \in S^{d} \mid \angle(x, y) \leq \rho\right\}
$$

denotes the spherical cap with center $x$ and radius $\rho$. For closed $M \subset S^{d}$ we may now define the spherical counterparts of inradius, circumradius and diameter. The spherical inradius $r^{\prime}(M)$ and the spherical incenter $c(M)$ are the radius and center, respectively, of a largest spherical cap $K_{r^{\prime}(M)}(c(M)) \subset M$. We remark that in contrast to the Euclidean case for convex $M$ the spherical incenter is always well defined. The spherical circumradius $R^{\prime}(M)$ and spherical circumcenter $C(M)$ are the radius and center, respectively, of the smallest spherical cap $K_{R^{\prime}(M)}(C(M)) \supset M$. Here the notions $R^{\prime}, r^{\prime}$ were chosen to distinguish the spherical circumradius and inradius from their Euclidean counterparts. For $M \subset S^{d}$ we denote the boundary of $K_{R^{\prime}(M)}(C(M))$ as the circumsphere of $M$. For any $M$ contained in a half-sphere the spherical diameter $D(M)$ is given by

$$
D(M)=\sup \{\angle(x, y) \mid x, y \in M\} .
$$

For $M \subset S^{d}, a_{1}, a_{2} \in S^{d}$ satisfying $a_{i}^{T} x \geq 0$ for all $x \in M, a_{i}^{T} x=0$ for at least one $x \in M$ and $a_{1} \neq a_{2}$ we denote by $\angle\left(a_{1}, a_{2}\right)$ a width of $M$. The breadth $d(M)$ is then defined as the minimum over all widths of $M$.

For $M \subset S^{d}$ the polar set $M^{*}$ is defined by

$$
M^{*}=\left\{x \in S^{d} \mid m^{T} x \geq 0 \text { for all } m \in M\right\} .
$$

In our context it is important to observe that the polar set of a spherical polyhedron is again a polyhedron and that inclusions are reversed, i.e. for $M \subset M^{\prime}$ we have $M^{*} \supset M^{\prime *}$. For $M=\left\{x^{1}, \ldots, x^{k}\right\}, x^{1}, \ldots, x^{k}$ linearly independent, we have that $M^{*}$ is a polyhedron with $k$ facets, which we denote as a $(k-1)$-cosimplex.

Further for $M \subset S^{d}$ convex we have the relations

$$
r^{\prime}(M)+R^{\prime}\left(M^{*}\right)=\pi, \quad d(M)+D\left(M^{*}\right)=2 \pi .
$$

In this terminology we have for an instance $A$ of $\mathrm{F}^{\prime}$ and set $Q=\left\{a_{j_{1}}, \ldots, a_{j_{l}}\right\}$ from Algorithm 2.6 with $P_{A} \neq \emptyset$ that $M=\operatorname{co} Q$ is an $(l-1)$-simplex with $M \subset P_{A}^{*}$ and 
$\cos \left(R^{\prime}(M)\right)=\operatorname{def} Q$. Correspondingly $M^{*}$ is an $(l-1)$-cosimplex with $P_{A} \subset M^{*}$ and $r^{\prime}\left(P_{A}\right) \leq r^{\prime}\left(M^{*}\right)=\pi-R^{\prime}(M)$.

Moreover, for $M \subset S^{d}$ the quantities $R^{\prime}(M)$ and $D(M)$ are not independent as the following theorem by Santaló [11] shows:

Theorem 3.1 (Santaló). Let $M \subset S^{d}$ be contained in a half-sphere. Then:

1. For $\cos R^{\prime} \geq 1 / \sqrt{d+1}$,

$$
\cos 2 R^{\prime} \leq \cos D \leq \frac{(d+1) \cos ^{2} R^{\prime}-1}{d} .
$$

2. For $0 \leq \cos R^{\prime} \leq 1 / \sqrt{d+1}$,

$$
\begin{aligned}
\cos 2 R^{\prime} \leq & \cos D \leq \frac{(d+1) \cos ^{2} R^{\prime}-1}{1+(d+1) \cos ^{2} R^{\prime}} \quad \text { for d odd }, \\
\cos 2 R^{\prime} \leq & \cos D \\
\leq & \frac{(d+1) \cos ^{2} R^{\prime}-1}{\left(1+(d+1) \cos ^{2} R^{\prime}\right)^{1 / 2}\left(1+(((d+1)(d-2)) /(d+2)) \cos ^{2} R^{\prime}\right)^{1 / 2}} \\
& \quad \text { for d even. }
\end{aligned}
$$

Using Santaló's theorem we may estimate the breadth $d\left(P_{A}\right)$ of $P_{A}$ by computing $R^{\prime}(Q)$. After a suitable transformation of coordinates we have for all $\left(x_{1}, \ldots, x_{d+1}\right)^{T} \in$ $P_{A}$ that $\left|x_{1}\right| \leq \sin d\left(P_{A}\right)$, i.e. all points of $P_{A}$ are close to the equator $\left\{\left(x_{1}, \ldots, x_{d+1}\right) \in\right.$ $\left.S^{d} \mid x_{1}=0\right\}$, and $P_{A}$ can be enlarged by a transformation. If we know that in the beginning $P_{A}$ is not too small, we must find a feasible $x^{k}$ after a number of steps, for otherwise the transformed $P_{A}$ would not fit on the sphere.

A suitable quantity to measure the size of $P_{A}$ is the volume and the estimate of the size of $P_{A}$ at the start of the algorithm is standard from the theory of linear programming. This leaves the determination of the hyperplane. While the proof of Santaló's theorem is constructive, the construction of the hyperplane needs the computation of about $\left(\begin{array}{c}d \\ d / 2\end{array}\right)$ distances and is therefore not practicable for a polynomial algorithm. Thus we substitute Santaló's theorem by a weaker one, for which we can easily compute all quantities.

Definition 3.2. Let $a_{1}, \ldots, a_{d+1} \in S^{d}$, such that $Q=\operatorname{co}\left\{a_{1}, \ldots, a_{d+1}\right\}$ is a spherical simplex. For each $a_{i}$ let $a_{i}^{\prime}$ be the intersection of the circle through $a_{i}$ and the circumcenter $C(Q)$ with $\operatorname{co}\left\{a_{1}, \ldots, a_{i-1}, a_{i+1}, \ldots, a_{d+1}\right\}$. Then the vertex diameter $D_{v}(Q)$ is defined by

$$
D_{v}(Q)=\max \left\{\angle\left(a_{1}, a_{1}^{\prime}\right), \ldots, \angle\left(a_{d+1}, a_{d+1}^{\prime}\right)\right\}
$$

Clearly the vertex diameter can be computed easily. While [11] contains no explicit estimate of the vertex diameter, the methods can be adopted. To be complete we present the changes in the proof. First we have for the vertex diameter of the regular simplex 
Lemma 3.3 (Santaló). Let $Q \subset S^{d}$ be a regular simplex with circumradius $R^{\prime}$ and vertex diameter $D_{v}$, then

$$
\cos D_{v}=\frac{(d+1) \cos ^{2} R^{\prime}-1}{\left(1+(d-1)(d+1) \cos ^{2} R^{\prime}\right)^{1 / 2}} .
$$

Proof. The formula is a simple combination of formulas (2.3) and (2.13) in [11].

Lemma 3.4 (Santaló). Let $Q=\operatorname{co}\left\{a_{1}, \ldots, a_{d+1}\right\} \subset S^{d}$ be a spherical simplex, such that all vertices are contained in its circumsphere. Let $C=\sum_{i=1}^{d+1} \mu_{i} a_{i}$ be the circumcenter of $Q$. Then for the circumradius $R^{\prime}$ of $Q$ it holds that

$$
\cos R^{\prime}=\left(\sum_{i=1}^{d+1} \mu_{i}\right)^{-1}, \quad \frac{1}{\cos ^{2} R^{\prime}} \leq(d+1) \sum_{i=1}^{d+1} \mu_{i}^{2} .
$$

Proof. We have

$$
\sum_{i=1}^{d+1} \mu_{i} \cos R^{\prime}=\sum_{i=1}^{d+1} \mu_{i} a_{i}^{T} C=C^{T} C=1
$$

From the equation we obtain

$$
\frac{1}{\cos ^{2} R^{\prime}}=\sum_{i=1}^{d+1} \mu_{i}^{2}+2 \sum_{1 \leq i<j \leq d+1} \mu_{i} \mu_{j} .
$$

The inequality is an immediate consequence of $2 \mu_{i} \mu_{j} \leq \mu_{i}^{2}+\mu_{j}^{2}$.

Lemma 3.5 (Santaló). Among all spherical d-simplices on $S^{d}$ with circumradius $R^{\prime}$ and $\cos R^{\prime}<1 / \sqrt{d+1}$, which contain all vertices in their circumsphere, the regular simplex has the minimal vertex diameter.

Proof. Assume that there is a simplex $Q \subset S^{d}$ with circumradius $R^{\prime}$, circumcenter $C$ and vertex diameter $\Delta_{v}$, which has all vertices on its circumsphere and has a smaller vertex diameter than the regular simplex with circumradius $R^{\prime}$. Let $D_{v}$ be the vertex diameter of the regular simplex with circumradius $R^{\prime}$. From (7) we have $\cos D_{v}<0$. Let $a_{1}, \ldots, a_{d+1}$ be the vertices of $Q$. We have

$$
C=\sum_{i=1}^{d+1} \mu_{i} a_{i} \quad \text { with } \quad \mu_{i}>0 .
$$

and

$$
a_{1}^{\prime}=\sum_{i=2}^{d+1} \mu_{i} a_{i} /\left\|\sum_{i=2}^{d+1} \mu_{i} a_{i}\right\|
$$


From this we obtain

$$
\begin{aligned}
\mu_{1} \cos R^{\prime} & =\mu_{1} a_{1}^{T} C \\
& =\mu_{1}^{2}+\mu_{1}\left(\sum_{i=2}^{d+1} \mu_{i}^{2}+2 \sum_{2 \leq i<j \leq d+1} \mu_{i} \mu_{j} a_{i}^{T} a_{j}\right)^{1 / 2} \cos \angle\left(a_{1}, a_{1}^{\prime}\right) .
\end{aligned}
$$

By our assumption we have $\cos \angle\left(a_{1}, a_{1}^{\prime}\right)>\cos D_{v}$. Thus (8) yields

$$
\mu_{1} \cos R^{\prime}-\mu_{1}^{2}-\mu_{1}\left(\sum_{i=2}^{d+1} \mu_{i}^{2}+2 \sum_{2 \leq i<j \leq d+1} \mu_{i} \mu_{j} a_{i}^{T} a_{j}\right)^{1 / 2} \cos D_{v}>0 .
$$

For $a_{2}, \ldots, a_{d+1}$ we obtain inequalities analogous to (9). Summing up all these inequalities, using the Cauchy-Schwarz inequality $x^{T} y \leq\|x\|^{1 / 2}\|y\|^{1 / 2}$ and taking $\|C\|=1$ into account we obtain

$$
\begin{aligned}
0< & \sum_{k=1}^{d+1} \mu_{k} \cos R^{\prime}-\sum_{k=1}^{d+1} \mu_{k}^{2} \\
& -\sum_{k=1}^{d+1} \mu_{k}\left(\sum_{i=1}^{d+1} \mu_{i}^{2}+2 \sum_{i \neq k} \sum_{i} \mu_{j} a_{i}^{T} a_{j}\right)^{\cos D_{v}} \\
< & \sum_{k=1}^{d+1} \mu_{k} \cos R^{\prime}-\sum_{k=1}^{d+1} \mu_{k}^{2}-\left(\sum_{k=1}^{d+1} \mu_{k}^{2}\right)^{1 / 2}\left(d \sum_{i=1}^{d+1} \mu_{i}^{2}+(d-1)\left(1-\sum_{i=1}^{d+1} \mu_{i}^{2}\right)\right)^{1 / 2} \\
& \times \sum_{k=1}^{d+1} \mu_{k} \cos D_{v} R^{\prime}-\sum_{k=1}^{d+1} \mu_{k}^{2}-\left(\sum_{k=1}^{d+1} \mu_{k}^{2}\right)^{1 / 2}\left(\sum_{i=1}^{d+1} \mu_{i}^{2}+(d-1)\right)^{1 / 2} \cos D_{v}
\end{aligned}
$$

Solving (10) for $\cos D_{v}$ and using the identity and inequality from Lemma 3.4 gives finally

$$
\begin{aligned}
\cos D_{v} & <\frac{1-\sum_{k=1}^{d+1} \mu_{k}^{2}}{\left(\sum_{k=1}^{d+1} \mu_{k}^{2}\right)^{1 / 2}\left(\sum_{i=1}^{d+1} \mu_{i}^{2}+(d-1)\right)^{1 / 2}} \\
& \leq \frac{(d+1) \cos ^{2} R^{\prime}-1}{\left(1+(d+1)(d-1) \cos ^{2} R^{\prime}\right)^{1 / 2}}
\end{aligned}
$$

This is a contradiction to (7).

By Lemmas 3.3 and 3.5 we may determine with the help of a set $Q$ two half-spheres, which contain $P_{A}$ in their intersection and the angle $\varphi$ between them is at most

$$
\cos \varphi \geq \frac{1-(d+1) \cos ^{2} R^{\prime}(Q)}{\left(1+(d-1)(d+1) \cos ^{2} R^{\prime}(Q)\right)^{1 / 2}} .
$$


Equation (11) yields, by an easy calculation and further estimation,

$$
\sin \varphi \leq(d+1) \cos R^{\prime} .
$$

Next we show that for $\varphi$ sufficiently small we may find a transformation of the sphere, which maps spherical polyhedra into spherical polyhedra and increases the volume of $P_{A}$ by a prescribed factor. Any spherical map

$$
B: S^{d} \rightarrow S^{d}, \quad B(x)=B^{\prime} x /\left\|B^{\prime} x\right\|
$$

with a nondegenerate linear map $B^{\prime}$ has the first property. The influence of $B$ on the volume is most easily studied by use of parameterizations.

A parameterized $d$-surface in $\mathbb{R}^{d+1}$ is a differentiable injective map $\Phi: G \subset \mathbb{R}^{d} \rightarrow$ $\mathbb{R}^{d+1}$ such that for every $x \in G$ the Jacobi-matrix $(\partial \Phi / \partial x)$ is nondegenerate. For $M \subset \Phi(G)$ the volume of $M$ is given by

$$
V(M)=\int_{\Phi^{-1}(M)} \operatorname{det} \Phi d x,
$$

where det $\Phi$ denotes the volume of the $d$-dimensional parallelepiped spanned by $\partial \Phi /$ $\partial x_{1}, \ldots, \partial \Phi / \partial x_{d}$. For a differentiable map $\psi: \mathbb{R}^{d+1} \rightarrow \mathbb{R}^{d+1}$ with appropriate properties, $\Psi \circ \Phi$ is a parameterized surface and consequently

$$
V(\Psi(M))=\int_{\Phi^{-1}(M)} \operatorname{det}(\Psi \circ \Phi) d x
$$

is the volume of $\Psi(M)$. Thus $(\operatorname{det}(\Psi \circ \Phi) / \operatorname{det} \Phi)\left(\Phi^{-1}(x)\right)$ is the local change of volume in $x \in \Phi(G)$ under the map $\Psi: \Phi(G) \rightarrow \mathbb{R}^{d+1}$.

Here we are interested in the case

$$
\begin{aligned}
& \Phi: B^{d} \rightarrow \mathbb{R}^{d+1}, \quad \Phi\left(x_{1}, \ldots, x_{d}\right)=\left(x_{1}, \ldots, x_{d}, \sqrt{1-\sum_{i=1}^{d} x_{i}^{2}}\right)^{T}, \\
& \Psi_{\alpha}: \mathbb{R}^{d+1} \rightarrow \mathbb{R}^{d+1}, \\
& \Psi_{\alpha}\left(x_{1}, \ldots, x_{d+1}\right)=\left(\alpha x_{1}, x_{2}, \ldots, x_{d+1}\right)^{T} / \sqrt{1+\left(\alpha^{2}-1\right) x_{1}^{2}}, \quad \alpha>0 .
\end{aligned}
$$

The restriction of $\Psi_{\alpha}$ on $S^{d}$ is the spherical transformation $\left(\alpha x_{1}, x_{2}, \ldots, x_{d+1}\right)^{T} /$ $\left\|\left(\alpha x_{1}, x_{2}, \ldots, x_{d+1}\right)^{T}\right\|$. We have chosen $\Psi_{\alpha}$ rather than the other transformation, as its derivatives can be computed more easily. In particular we consider the point $\bar{x}=$ $\left(\beta, 0, \ldots, 0, \sqrt{1-\beta^{2}}\right)^{T}$ with $0 \leq \beta<1$. Computation of the Jacobi matrices and of the determinants with the help of Gram determinants yields

$$
\operatorname{det} \Phi(\bar{x})=\frac{1}{\left(1-\beta^{2}\right)^{1 / 2}}, \quad \operatorname{det}\left(\Psi_{\alpha} \circ \Phi\right)(\bar{x})=\frac{\alpha}{\left(1-\beta^{2}\right)^{1 / 2}\left(1+\beta^{2}\left(\alpha^{2}-1\right)\right)^{(d+1) / 2}} .
$$

Thus the transformation $\psi_{\alpha}$ gives for all $\left(x_{1}, \ldots, x_{d+1}\right)^{T} \in S^{d}$ with $x_{1}=\beta$ the local change of volume

$$
f(\beta)=\frac{\alpha}{\left(1+\beta^{2}\left(\alpha^{2}-1\right)\right)^{(d+1) / 2}} .
$$


Consequently we obtain for $M \subset S^{d}$ with $x_{1} \leq \beta$ for all $\left(x_{1}, \ldots, x_{d+1}\right)^{T} \in M$ and $\alpha>0$ the estimate

$$
V\left(\Psi_{\alpha}(M)\right) \geq \frac{\alpha}{\left(1+\beta^{2}\left(\alpha^{2}-1\right)\right)^{(d+1) / 2}} V(M) .
$$

We may summarize the previous considerations in

Lemma 3.6. Let $M \subset S^{d}$ such that for all $\left(x_{1}, \ldots, x_{d+1}\right)^{T} \in M$ it holds that $x_{1} \leq \beta$. Further, let $\Psi_{\alpha}: S^{d} \rightarrow S^{d}, \alpha>0$, be given by (14). Then the volume of $\Psi_{\alpha}(M)$ can be estimated by (15).

Evidently we may generalize the transformation $\Psi_{\alpha}$ to the case of a transformation orthogonal to an arbitrary equator $a^{T} x=0$ instead of the equator $x_{1}=0$.

As we work with the homogenized problem, we have to study the influence of (3) on the volume. Using the same technique as before we find for $M \subset \mathbb{R}^{d}$ for the volume of the homogenized set

$$
V(\Phi(M))=\int_{M} \frac{1}{\left(\|x\|^{2}+1\right)^{(d+1) / 2}} d x .
$$

Thus we have

Lemma 3.7. Let $M \subset \mathbb{R}^{d}$ with $\|x\| \leq R$ for all $x \in M$. Then $V(\Phi(M)) \geq(R+$ $1)^{-(d+1)} V(M)$.

The following considerations concerning polynomial algorithms could be done in the context of oracle-polynomial time algorithms for convex bodies in [5]. The presentation becomes somewhat simpler if we follow the presentation in [9] for algorithms for the feasibility problem of linear systems.

According to the corollary to Lemma 8.7 in [9] we can solve F in polynomial time, if we can solve the following problem in polynomial time: For the set

$$
M=\left\{x \in \mathbb{R}^{d} \mid a_{i}^{T} x>b_{i}, i=1, \ldots, n\right\}
$$

of size $L$ find a point in $M$ or show that there exists no such point in time polynomial in $L$. Here size $L$ of the system measures the amount of space needed to write down the inequalities. For a precise definition of the size see [5] or [9].

We have that either $M$ is empty or the volume of $M$ is not too small:

Lemma 3.8. If the set $M$ from (16) is nonempty, then

$$
V\left(M \cap\left\{x \mid\|x\| \leq d 2^{L}\right\}\right) \geq 2^{-(d+2) L} .
$$

Proof. Lemma 8.14 of [9].

For the $a_{i}, b_{i}$ from (16) let $a_{i}^{\prime}=\left(a_{i}^{T},-b_{i}\right)^{T}$. Then we have for the homogenization of $M$,

$$
\Phi(M)=\left\{x \in S^{d} \mid a_{i}^{\prime T} x>0, i=1, \ldots, n, x_{d+1}>0\right\} .
$$


We may combine Lemmas 3.7 and 3.8 to obtain

Lemma 3.9. Let $M$ be the set from (16) with size L. Then

$$
V(\Phi(M)) \geq 2^{-3(d+2) L}
$$

or $\Phi(M)$ is empty.

Now we have collected the parts to show that the following modification of Algorithm 2.8 is polynomial.

\section{Algorithm 3.10.}

Input: A linear feasibility problem (1), such that the set of feasible points is either empty or contains interior points.

Output: A feasible point $x$ or the assertion that such a point does not exist.

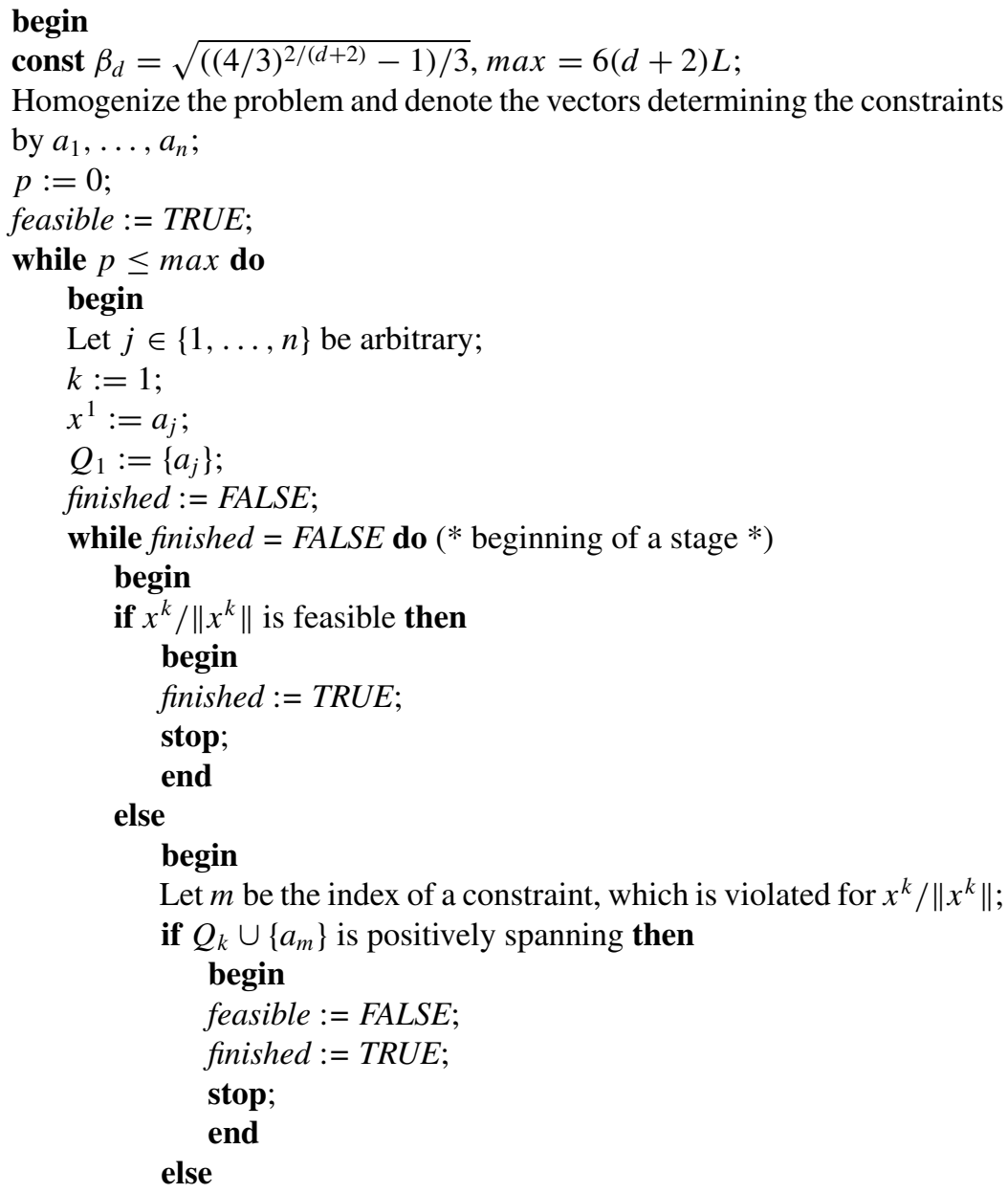




\section{begin}

Let $y$ be the point on $\overline{x^{k} a_{m}}$ closest to the origin;

Let $C$ be the center of the touching sphere of $Q_{k} \cup\left\{a_{m}\right\}$;

while $C \notin \operatorname{conv}\left(Q_{k} \cup\left\{a_{m}\right\}\right)$ do

begin

Let $y$ be the point, where the line segment $\overline{y C}$ intersects

the relative boundary of $\operatorname{conv}\left(Q_{k} \cup\left\{a_{m}\right\}\right)$;

Let $F$ be a facet of $\operatorname{conv}\left(Q_{k} \cup\left\{a_{m}\right\}\right)$, which contains $y$;

Let $a_{j}$ be the point of $Q_{k}$, which is not contained in $F$;

$Q_{k}:=Q_{k} \backslash\left\{a_{j}\right\}$;

end;

$k:=k+1$;

$x^{k}:=C$;

$Q_{k}:=Q_{k-1} \cup\left\{a_{m}\right\} ;$

if def $Q_{k}<\beta_{d} /(d+1)$ then

begin

finished := TRUE;

$p:=p+1$;

Transform;

end;

end;

end;

end;

end;

end.

procedureTransform

begin

Determine the vertex diameter of $\operatorname{co} Q_{k}$ and let $a_{i}$ be the vertex which gives the vertex diameter;

Apply the transformation $\Psi_{2}$ with respect to the equator $a_{i}^{T} x=0$.

end.

Theorem 3.11. Algorithm 3.10 solves $F$ in polynomial time.

Proof. We denote by $M_{p}$ the set of feasible points before the $p$ th transformation. First we show the correctness of the algorithm. If $x^{k} /\left\|x^{k}\right\|$ is feasible, then the algorithm trivially gives the correct result. If we find that $Q_{k} \cup\left\{a_{m}\right\}$ is positively spanning, then $M \subset P_{Q_{k}} . P_{Q_{k}}$ is a lower-dimensional sphere and thus $V\left(P_{Q_{k}}\right)=0$ and we have by our assumption that the instance is infeasible (see Lemma 3.9).

Now we consider the state of the algorithm at the call of procedure Transform. We have $\cos R^{\prime}\left(Q_{k}\right) \leq \beta_{d} /(d+1)$. By (12) we find that all points of $M_{p}$ are contained in the zone $\left\{x \in S^{d} \mid 0 \leq a_{i}^{T} x \leq \beta_{d}\right\}$ for the $a_{i}$ giving the vertex diameter. From this we obtain by Lemma 3.6 that $V\left(M_{p+1}\right) / V\left(M_{p}\right) \geq \frac{3}{2}$. Finally we obtain by Lemma 3.9 that after max steps we have $V\left(M_{\max }\right) \geq 2>V\left(S^{d}\right)$. Consequently, an existing feasible point must be found before this step. This proves the correctness of the algorithm. 
To calculate the number of computations we only have to estimate the number of iterations in a stage. By a power series expansion we see easily that $\beta \geq c d^{-1 / 2}$ for suitable $c>0$. Thus by Lemma 2.10 there are $O\left(d^{3}\right)$ iterations.

It remains to show that it is sufficient to do all calculations with rational numbers whose size is bounded by a polynomial in $L$. We must show that by some rounding process the number of steps is not increased too much. While the necessary steps are not very difficult the calculations tend to become rather longish if one wants to work out the precise number of steps.

Thus we only indicate that there are no particular difficulties and skip all the details. We work with unnormalized constraints. In this way the homogenization is just to replace the inequality $a_{i}^{T} x \geq b_{i}$ by $\left(a_{i}^{T}, b_{i}\right) x \geq 0$. We denote the inner while-loop as a stage of the algorithm. During a stage we essentially have to compute the centers $C$ of touching spheres. We have $C=\sum_{a_{i} \in Q_{k}} \lambda_{j} a_{j}$ and $C^{T} a_{i}=R\left\|a_{i}\right\|, a_{i} \in Q_{k}$, for the radius $R$ of the touching sphere. Thus we have to solve a sequence of linear equations, which is a polynomial operation in the size of the encoding length of the input. As (5) which controls the progress of the algorithm is independent of the encoding length, we may round the quantities $R\left\|a_{i}\right\|$ in a suitable way such that a stage still works and (5) remains essentially valid.

In each stage the $a_{i}$ are changed by the procedure Transform. Calculating with unnormalized integer vectors $a_{i}$ and $\alpha=2$ the transformation becomes

$$
a_{i}:=a_{i}+a_{j} a_{j}^{T} a_{i}, \quad i=1, \ldots, n,
$$

for a suitable $j$. In this way the encoding length may increase exponentially. Using a simple rounding process we obtain from $a_{j}$ a new vector $\bar{a}_{j}$ such that for a suitable constant $\gamma$ we have

$$
\max _{k}\left(\bar{a}_{j k}\right) \leq \gamma d
$$

and

$$
\angle\left(a_{j}, \bar{a}_{j}\right)<1 /(2 d)
$$

We replace (17) by

$$
a_{i}:=a_{i}+\bar{a}_{j} \bar{a}_{j}^{T} a_{i}, \quad i=1, \ldots, n,
$$

for a suitable $j$. If we replace the stopping condition $\operatorname{def} Q_{k}<\beta_{d} /(d+1)$ by def $Q_{k}<$ $\beta_{d} /(2 d+2)$ we find that the algorithm remains correct and the encoding length of the $a_{i}$ grows in a polynomial way.

Some remarks concerning the algorithm:

1. If we compare Algorithms 2.8 and 3.10 we find that we have replaced the condition in Algorithm 2.8 that the new $a_{m}$ is given by a most violated constraint by the weaker condition that the new $a_{m}$ in Algorithm 3.10 is given by any violated constraint. We can easily check that it is sufficient to choose any $a \neq 0$, such that $a^{T} x^{k}<0$, but $a^{T} x \geq 0$ for all $x \in P_{A}$, i.e. if $\left\{a^{T} x=0\right\}$ is a separating great sphere for $x^{k}$ and $P_{A}$. Moreover, we made no use of the fact that $P_{A}$ is a polyhedron, but used only that the volume of $P_{A}$ cannot 
be too small. Now a separating hyperplane for the unhomogenized problem immediately yields a separating great sphere for the homogenized problem. Thus the algorithm is essentially a polynomial algorithm for the following convex feasibility problem: Given is a convex set $K \in \mathbb{R}^{d}$ which is either empty or well bounded in the language of [5] and an oracle which for any $x \in \mathbb{R}^{d}$ confirms that $x \in K$ or gives a separating hyperplane. Under these assumptions find a point in $K$ or show that $K$ is empty.

2. While the previous remark pointed out that from a theoretical point of view it is sufficient to choose any separating great sphere, from a practical point of view this is certainly not the case. This is shown by the following example, which shows that the inner loop in Algorithm 3.10 may need many iterations:

In this example the sets $Q_{k}$ always have three elements which are for simplicity unnormalized. Let

$$
Q_{k}=\left\{(1, \varepsilon, 0, \ldots, 0)^{T},(-1, \varepsilon, 0, \ldots, 0)^{T},\left(0,-\delta_{k}, 1,0, \ldots, 0\right)^{T}\right\},
$$

where $\varepsilon$ and $\delta_{k}$ are positive numbers of the kind that $\delta_{k}$ is small with respect to 1 , and $\varepsilon$ is small with respect to $\delta_{k}$. The points $x^{k}$ are of the form $x^{k}=\left(0,1, \eta_{k}, 0, \ldots, 0\right)^{T}$. $\left\{\left(0,-\delta_{k+1}, 1,0, \ldots, 0\right)^{T} x=0\right\}$ is a separating great sphere if $\delta_{k+1}>\eta_{k}$. The computation of $x^{k+1}$ gives

$$
x^{k+1}=\left(0,1, \eta_{k+1}, 0, \ldots, 0\right)^{T}, \quad \eta_{k+1}=\delta_{k+1}+\varepsilon \frac{\sqrt{1+\delta_{k+1}^{2}}}{\sqrt{1+\varepsilon^{2}}} .
$$

Thus if $\delta_{k+1}$ is close to $\eta_{k}$ we have that the distance $\angle\left(x^{k}, x^{k+1}\right)$ is of order $\varepsilon$. Now the iteration may be repeated with a $\delta_{k+2}$ which is of the same order as $\delta_{k+1}$. This behavior does not affect the polynomiality as $\varepsilon$ is bounded below by a function depending on the dimension, but demonstrates that the convergence may be rather slow.

3. If we choose the most violated constraint, then this phenomenon cannot occur, as is shown by the discussion after Lemma 2.10 .

4. While the choice of $x^{k}$ as the circumcenter of the sets $Q_{k}$ is fundamental for our work, this is certainly not the case for the construction of the transformation. Although it did secure the polynomiality of the algorithm, there are some apparent disadvantages. We have to work with three different quantities (circumradius, vertex diameter, volume) and these quantities are rather weakly related. Moreover, the volume does not behave very nicely for transformations of the sphere. The apparent choice for a transformation would be to transform after each iteration co $Q_{k}$ into a regular simplex of the same circumradius such that the circumcenter of co $Q_{k}$ is transformed to the circumcenter of the resulting regular simplex. Unfortunately we do not know of any quantity which behaves nicely under this transformation and thus do not even know whether the resulting algorithm is convergent.

5. Still there are probably other transformations which are easier to handle than the one sketched above and lead to polynomial algorithms. One approach might be the following: We want to construct in polynomial time a positively spanning set for infeasible problems. For our heuristic considerations we assume that this positively spanning set is actually a positive basis. As we want to construct a positive basis, we look at the violation. Considering the fact that for a regular simplex the quotient of the 
circumradius and the inradius is $d$ we find that $v\left(x^{k}\right) \geq 1 /(d+1)$ if the points of the positive basis form a regular simplex. Now, let us assume that we have found a point $x^{k}$ such that $v\left(x^{k}\right)$ is small compared with $1 /(d+1)$. Let $Q$ be an arbitrary positive basis spanned by the $a_{i}$. Then it is easy to see that there must be a face $F$ of conv $Q$, such that $F$ is nearly parallel to $\left\{\left(x^{k}\right)^{T} x=0\right\}$ and has a small distance from the origin. We apply for suitable $\alpha>1$ a transformation $\Psi_{\alpha}$ with respect to the equator $\left\{\left(x^{k}\right)^{T} x=0\right\}$. Locally we increase the violation. Globally it may be seen that the distance to the origin of all faces of conv $Q$, which have a dimension less than or equal to the dimension of $F$, is increased. One may hope that the minimal rate of increase is given by a function, which depends only on the dimension. Thus one should obtain fast, i.e. polynomial, convergence.

We have not tried to work out the theoretical details of this algorithm. Thus we do not know whether it works and have no information on its theoretical quality. We may say that this algorithm would avoid the problems of our algorithm which we pointed out in the previous remark, as in our context it is certainly more natural to deal with distances than with volume. Moreover, the transformation with a pole $x^{k}$ is more natural than the transformation with a pole $a_{i}$ and it is easy to implement this algorithm. This was in fact done with a transformation taking place whenever $v\left(x^{k}\right) \leq 1 / \sqrt{d}$. The surprisingly good results of this algorithm are presented in the next section.

Altogether we may say that solely as a consequence of the weak estimate in Lemma 2.10 it is possible to construct a sequence of transformations which transform the combinatorial Algorithm 2.8 into a polynomial one. This is a remarkable contrast to the combinatorial simplex algorithm, as for the simplex algorithm it is even unknown whether an optimal choice of pivot steps would lead to a polynomial algorithm (Hirsch conjecture, for an account see [13]).

Moreover, there is some hope that Algorithm 2.6 is polynomial. That would be of particular interest, as an algorithm which is combinatorial as well as polynomial might be a candidate for a strongly polynomial algorithm for F. It is still an open question whether such an algorithm exists. For details about this problem see [5].

\section{Implementation of the Algorithms and Numerical Experiments}

Up to now we have only studied the geometry of the algorithms and we still have the problem of finding a practical way to perform the necessary calculations. We do not want to go into details or find the optimal way to do this but just show that there are no particular problems and that the complexity of a single step is on average comparable with the simplex algorithm, i.e. $O(d n)$.

The only steps in Algorithm 2.6 where the computations are not completely obvious are where we compute the center $C$ of the touching sphere and check whether it is in the convex hull of the $a_{i}$. We observe that by our construction $Q_{k}$ is linearly independent and $Q_{k} \cup\left\{a_{m}\right\}$ is affinely independent. We compute a least squares representation

$$
a_{m}=a+\sum_{a_{i} \in Q_{k}} \lambda_{i} a_{i}, \quad a \text { orthogonal to all } \quad a_{i} \in Q_{k},
$$


and distinguish two cases:

1. $a=0$. Then $Q_{k} \cup\left\{a_{m}\right\}$ is linearly dependent, $C=0$ and using (19) we easily find a representation of the origin as an affine combination of $Q_{k} \cup\left\{a_{m}\right\}$.

2. $a \neq 0$. We observe that $C$ is characterized by the following three properties:

(a) $a_{i}^{T} C=a_{j}^{T} C>0$ for all $a_{i}, a_{j} \in Q_{k} \cup\left\{a_{m}\right\}$.

(b) $C=\sum_{a_{i} \in Q_{k} \cup\left\{a_{m}\right\}} \mu_{i} a_{i}$ with $\mu_{i} \in \mathbb{R}$.

(c) $\sum \mu_{i}=1$.

Thus if a $C^{\prime} \neq 0$ has the first two properties, then $C$ is just a multiple of $C^{\prime}$.

We look at the points $y$ constructed in the algorithm. We find inductively that we have

$$
\begin{aligned}
\angle\left(y, a_{i}\right) & =\angle\left(y, a_{l}\right) \leq \angle\left(y, a_{m}\right) \quad \text { for all } \quad a_{i}, a_{l} \in Q_{k}, \\
y & =\sum_{a_{i} \in Q_{k} \cup\left\{a_{m}\right\}} \mu_{i} a_{i}, \quad \text { with all } \quad \mu_{i} \geq 0 .
\end{aligned}
$$

Thus using (19) we find that there is a $C^{\prime}$ of the form $C=y+\rho a$ for suitable $\rho>0$. From this we determine $C$ as an affine combination of $Q_{k} \cup\left\{a_{m}\right\}$.

The next $a_{i}$ to leave $Q_{k}$ is then apparently found by a simple line search. It remains to solve (19). To do this we consider the $Q_{k}$ as matrices with columns $a_{i}$. We observe that consecutive matrices $Q_{k}, Q_{k+1}$ only differ in that a column is added or taken away. If we solve (19) by computing a QR-factorization of $Q_{k}$, then it is well known that the QRfactorizations of consecutive $Q_{k}, Q_{k+1}$ can be obtained by an updating process which takes only $O\left(d^{2}\right)$ computations, see [4]. Thus taking into account that the determination of $a_{m}$ in step 2 takes $O(d n)$ computations and we have on average to add one column to $Q_{k}$ and take one column away, we find that a step of Algorithm 2.6 takes $O(d n)$ computations on average.

Clearly our results above give no information whether the algorithms could be of practical use. An obvious way to test this would be to solve the test problems as provided by http://www.netlib.org/lp. These problems are sparse optimization problems, i.e. to solve them we have to deal with many issues which are rather unrelated to the basic algorithm, but will strongly influence the results. Among these are solving the optimization problem by an algorithm for feasibility, dealing with equality constraints, dealing with the sparsity. Thus we have taken a much more simple approach. We generated three different sets of problems. The most basic way was done by generating in a random way $n$ vectors of unit length $a_{i}$ and $n$ negative constants $b_{i}$ (Ex1 in the following tables). Thus we obtain a system of inequalities $a_{i}^{T} x \geq b_{i}$ which has the origin in the interior of the feasible region. In the next set of problems we chose $b_{i}=0$ for $i=1, \ldots, d+1$ and $a_{d+1}=-\sum_{i=1}^{d} a_{i} /\left\|\sum_{i=1}^{d} a_{i}\right\|$. Thus in this case we obtained with probability 1 problems which have exactly one feasible point (Ex2). The last set differed from the second only in that we chose $b_{d+1}=10$ to construct infeasible problems (Ex3). In order to use the origin as a starting point all systems were translated by a random vector.

If we look at the problems then we see that after choosing the correct set of indices the second problem is equivalent to the solution of a system of linear equations. If we use the Gauss algorithm, and allow it to transform the complete problem in every step, then we see that a solution would involve $d$ steps, each with $O(n d)$ operations. In particular, the number of steps would be independent of the number of inequalities. Certainly we 
would consider such an algorithm as excellent from a practical point of view and we may ask how close we can get.

We used three different algorithms for the solution of the problems. First we used the following variant of the simplex algorithm to have a basis of comparison. We consider the problem as the dual of a problem in standard form. We perform $d$ pivot steps to generate a basis. This corresponds to asking for equality in the inequalities corresponding to the basic columns. Then we choose a positive constant right side and use the simplex algorithm to solve the generated problem. If the algorithm stops with a finite solution, this shows the existence of a feasible point in the dual problem in the intersection of the constraints corresponding to the basic columns. If the algorithm stops with unboundedness this shows the infeasibility of the dual problem. This algorithm is denoted Alg 1 in the following tables.

The next algorithm was a straightforward implementation of Algorithm 2.6 (Alg 2). The last experimental algorithm (Alg 3) used an additional rescaling in Algorithm 2.6. Here it does not make much sense to use the polynomial Algorithm 3.10 as the number of steps before the first transformation takes place is not much smaller than the total number of steps for the complete solution by Algorithm 2.6. Instead we used the following procedure. We have by (5) that every step of the algorithm makes relative progress of at least $\sqrt{1-v\left(x^{k}\right)^{2}}$. Thus when $v\left(x^{k}\right)$ becomes less than $1 / \sqrt{d}$ then we do the following. Let $v\left(x^{k}\right)$ be determined by the constraint $a_{r}$, i.e. $v\left(x^{k}\right)=-a_{r}^{T} x^{k} /\left\|x^{k}\right\|$. We set $a_{i}^{\prime}=\left(I+\lambda x^{k} x^{k^{T}}\right) a_{i}, i=1, \ldots, n$, and renormalize the $a_{i}^{\prime}$ to unit length. Here $\lambda$ is chosen in a way such that $a_{r}^{\prime} x^{k} /\left\|x^{k}\right\|=-\sqrt{2 / d}$. We observe that the matrix $Q_{k}$ is transformed by the formula $Q_{k}=\mu\left(Q_{k}+w x^{k^{T}}\right)$ for a scalar $\mu$ and a vector $w$ which are easily determined. For such a transformation it is possible to update the QRfactorization in $O\left(d^{2}\right)$ computations, see [4]. Thus the transformation takes a total of $O(n d)$ computations.

To test the dependence on the dimension we chose the dimensions $d=10,20,40$, $80,160,320$ and 640 and $8 d$ inequalities for each dimension. To test the dependence on the number of inequalities we chose $d=100$ and $n=400,800,1600,3200,6400$. For each case five examples were generated and solved. The average numbers of steps are shown in Tables 1 and 2.

In the tables the second column for the third algorithm gives the number of rescalings. Moreover, the numbers for the simplex algorithm do not give the $d$ steps necessary to obtain the starting tableau. We see that all algorithms show little dependence on the number of constraints, in particular for Alg 3 this dependence appears to be sublogarithmic.

For the dependence on $d$ we may not expect spectacular results, as we know by the remarks at the beginning that we may not hope for less than $d$ iterations but on the other hand the simplex algorithm solved all examples in less than $12 d$ iterations. However, we see that for large dimensions the new algorithms need fewer iterations and in particular Alg 3, the algorithm with the rescaling, did very well.

To obtain some more insight into the dependence on the dimension we did a best fit of the form $\alpha d^{\beta}$ for all series and all algorithms. Here we computed two values for the simplex algorithm, for the first value we counted the $d$ steps for the initialization while in the second value these steps were neglected. For the algorithm with the rescaling we did not take into account the numbers of rescalings, as this number appears to have little dependence on the dimension. The results are presented in Table 3 . 
Table 1. Number of steps in dependence of the dimension.

\begin{tabular}{rrrrrr}
\hline & Dim & Alg 1 & Alg 2 & \multicolumn{2}{c}{ Alg 3 } \\
\hline Ex1 & 10 & 13.4 & 20.6 & 16.8 & 5.8 \\
& 20 & 31.2 & 57.8 & 35.2 & 12.0 \\
& 40 & 93.4 & 146.0 & 69.2 & 18.8 \\
& 80 & 274.4 & 353.2 & 146.6 & 23.2 \\
& 160 & 769.2 & 926.0 & 294.8 & 27.4 \\
& 320 & 2114.6 & 2156.6 & 585.0 & 29.8 \\
& 640 & 6321.0 & 4756.4 & 1179.0 & 33.8 \\
Ex2 & 10 & 17.0 & 28.8 & 25.8 & 9.2 \\
& 20 & 42.6 & 62.6 & 54.2 & 14.0 \\
& 40 & 122.0 & 154.8 & 108.8 & 20.8 \\
& 80 & 346.6 & 385.2 & 228.8 & 29.6 \\
& 160 & 925.0 & 923.8 & 528.2 & 35.0 \\
& 320 & 2528.0 & 2296.8 & 939.0 & 41.2 \\
Ex3 & 640 & 7294.4 & 5388.0 & 1909.4 & 45.4 \\
& 10 & 15.8 & 27.4 & 24.0 & 7.4 \\
& 20 & 41.0 & 58.4 & 50.2 & 11.0 \\
& 40 & 112.4 & 141.2 & 101.2 & 17.0 \\
& 80 & 326.2 & 368.6 & 210.0 & 19.8 \\
& 160 & 887.0 & 857.2 & 422.0 & 21.0 \\
& 320 & 2565.2 & 2183.2 & 867.0 & 22.6 \\
& 640 & 7151.0 & 5125.2 & 1787.0 & 23.4 \\
\hline
\end{tabular}

Table 2. Number of steps in dependence of the number of inequalities.

\begin{tabular}{lrrrrr}
\hline & \multicolumn{1}{c}{$n$} & Alg 1 & Alg 2 & \multicolumn{2}{c}{ Alg 3 } \\
\hline Ex1 & 400 & 222.0 & 336.4 & 158.2 & 20.8 \\
& 800 & 386.6 & 481.0 & 185.8 & 24.4 \\
& 1600 & 502.0 & 597.2 & 210.8 & 27.6 \\
& 3200 & 644.6 & 670.2 & 225.4 & 28.6 \\
& 6400 & 745.2 & 781.4 & 244.2 & 30.2 \\
Ex2 & 400 & 302.4 & 345.0 & 247.2 & 27.6 \\
& 800 & 456.6 & 536.0 & 294.6 & 32.2 \\
& 1600 & 570.6 & 602.8 & 312.2 & 32.8 \\
& 3200 & 735.0 & 713.4 & 330.0 & 34.6 \\
& 6400 & 869.6 & 794.2 & 360.0 & 38.0 \\
Ex3 & 400 & 297.4 & 334.0 & 224.6 & 17.8 \\
& 800 & 449.8 & 491.8 & 261.0 & 19.8 \\
& 1600 & 553.8 & 545.6 & 279.2 & 21.0 \\
& 3200 & 732.6 & 657.8 & 293.6 & 20.6 \\
& 6400 & 838.6 & 699.4 & 304.2 & 20.8 \\
\hline
\end{tabular}

Table 3. Rate of growth.

\begin{tabular}{|c|c|c|c|c|c|c|c|c|}
\hline & \multicolumn{4}{|c|}{ Alg 1} & \multicolumn{2}{|c|}{ Alg 2} & \multicolumn{2}{|c|}{ Alg 3} \\
\hline & $\alpha$ & $\beta$ & $\alpha$ & $\beta$ & $\alpha$ & $\beta$ & $\alpha$ & $\beta$ \\
\hline Ex1 & 0.8735 & 1.3783 & 0.3890 & 1.4990 & 1.1122 & 1.3093 & 1.6269 & 1.0214 \\
\hline Ex2 & 1.0709 & 1.3432 & 0.5605 & 1.4621 & 1.4575 & 1.2719 & 2.4228 & 1.0334 \\
\hline Ex3 & 0.9950 & 1.3793 & 0.5010 & 1.4779 & 1.3538 & 1.2747 & 2.2439 & 1.0334 \\
\hline
\end{tabular}


We find that the simplex algorithm and Algorithm 2.6 are superlinear, where the exponent of Algorithm 2.6 is slightly smaller for all series, while the experimental algorithm is very close to being linear for our examples and thus for these examples very close to the hypothetical Gauss-type algorithm.

Altogether the examples show that the concepts developed here might be of practical interest.

\section{Acknowledgment}

The author thanks M. Henk and A. Schürmann for valuable discussions, hints to the literature and reading a previous version of this paper, moreover, he thanks an unknown referee for suggesting numerous improvements for a previous version of this paper.

\section{References}

1. Agmon, S.: The relaxation method for linear inequalities, Canad. J. Math. 6 (1954), 382-392.

2. Bauschke, H.H.; Borwein, J.M.: On projection algorithms for solving convex feasibility problems, SIAM Rev. 38 (1996), 367-424

3. Flåm, S.D.; Zowe, J.: Relaxed outer projections, weighted averages and convex feasibility, BIT 30 (1990), 289-300.

4. Golub, G.H.; Van Loan, C.F.: Matrix Computations, North Oxford Academic, Oxford, 1983.

5. Grötschel, M.; Lovász, L.; Schrijver, A.: Geometric Algorithms and Combinatorial Optimization, SpringerVerlag, Berlin, 1988.

6. Karmarkar, N.: A new polynomial-time algorithm for linear programming, Combinatorica 4 (1984), 373-395.

7. Khachiyan, L.G.: A new polynomial algorithm in linear programming (Russian), Dokl. Akad. Nauk SSSR 244 (1979), 1093-1096 (English translation: Soviet Math. Dokl. 20 (1979), 191-194).

8. Motzkin, T.S.; Schoenberg, I.J.: The relaxation method for linear inequalities, Canad. J. Math. 6 (1954), 393-404.

9. Papadimitriou, C.H.; Steiglitz, K.: Combinatorial Optimization, Prentice-Hall, Englewood Cliffs, New Jersey, 1982.

10. Potra, F.A.; Wright, S.J.: Interior-point methods, J. Comput. Appl. Math. 124 (2000), 281-302.

11. Santaló, L. A.: Convex regions on the $n$-dimensional spherical surface, Ann. of Math. 47 (1946), 448-459.

12. Schrijver, A.: Theory of Integer and Linear Programming, Wiley, Chichester, 1986.

13. Ziegler, G.: Lectures on Polytopes, Springer-Verlag, New York, 1994.

Received May 10, 2002, and in revised form August 19, 2003. Online publication May 26, 2004. 